\title{
The Exoscope versus operating microscope in microvascular surgery: A simulation non-inferiority trial
}

\author{
Georgios Pafitanis ${ }^{1,2,3}$, Michalis Hadjiandreou ${ }^{3}$, Alexander Alamri ${ }^{4}$, Christopher Uff ${ }^{4}$, \\ Daniel Walsh ${ }^{5}$, Simon Myers ${ }^{1,4}$ \\ ${ }^{1}$ Group for Academic Plastic Surgery, Microvascular Anastomosis Simulation Hub, The Blizard Institute, Queen Mary University of London, \\ London; ${ }^{2}$ Department of Plastic Surgery, Great Ormond Street Hospital for Children Foundation Trust, London; ${ }^{3}$ Barts' and The London \\ School of Medicine and Dentistry, London; ${ }^{4}$ Department of Neurosurgery, The Royal London Hospital, Barts Health NHS Trust, London; \\ ${ }^{5}$ Department of Neurosurgery, King's College Hospital, London, UK
}

Background The Exoscope is a novel high-definition digital camera system. There is limited evidence signifying the use of exoscopic devices in microsurgery. This trial objectively assesses the effects of the use of the Exoscope as an alternative to the standard operating microscope (OM) on the performance of experts in a simulated microvascular anastomosis.

Methods Modus V Exoscope and OM were used by expert microsurgeons to perform standardized tasks. Hand-motion analyzer measured the total pathlength (TP), total movements (TM), total time (TT), and quality of end-product anastomosis. A clinical margin of $\Pi$ was performed to prove non-inferiority. An expert performed consecutive microvascular anastomoses to provide the exoscopic learning curve until reached plateau in $\pi$.

Results Ten micro sutures and 10 anastomoses were performed. Analysis demonstrated statistically significant differences in performing micro sutures for TP, TM, and TT. There was statistical significance in TM and $\Pi$, however, marginal non-significant difference in TP regarding microvascular anastomoses performance. The intimal suture line analysis demonstrated no statistically significant differences. Non-inferiority results based on clinical inferiority margin $(\Delta)$ of $\Pi=10$ minutes demonstrated an absolute difference of 0.07 minutes between $\mathrm{OM}$ and Exoscope cohorts. A 51\%, 58\%, and 46\% improvement or reduction was achieved in $\Pi, T M, T P$, respectively, during the exoscopic microvascular anastomosis learning curve.

Conclusions This study demonstrated that experts' Exoscope anastomoses appear non-inferior to the $\mathrm{OM}$ anastomoses. Exoscopic microvascular anastomosis was more time consuming but end-product (patency) in not clinically inferior. Experts' "warm-up" learning curve is steep but swift and may prove to reach clinical equality.

Keywords Exoscope / Video telescope operating monitor / Microsurgery / Microscopy / Microvascular anastomosis
Correspondence: Georgios Pafitanis Group for Academic Plastic Surgery, Microvascular Anastomosis

Simulation Hub, The Blizard Institute, Queen Mary University of London, 4 Newark Street, Whitechapel, London E12AT, UK

Tel: $+44-2078827173$

Fax: +44-2078827172

E-mail: g.pafitanis@qmul.ac.uk

The authors would like to express their appreciation to lan Weighell and Chris Fell from Synaptive Medical Inc., for their contribution in providing the Modus V device. 


\section{INTRODUCTION}

In the early 1960s, the operating microscope (OM) provided magnification and illumination, and microsurgery was born [13]. Developments in digital imaging allowed surgeons in other areas to operate using scopes guided by a two-dimensional (2D) surgical field without magnification. Alternatives to the classical OM (Table 1) [4-8], have since anecdotally included a three-dimensional (3D) stereoscopic monitor system combined with the $\mathrm{OM}$ for head and neck free microvascular reconstructive procedures [9], and an exoscopic device excluding an OM in free autologous breast reconstruction [10].

The term "Exoscope" is derived from the Greek words "exo" (out of) + skopein (to look), and it provides a high-definition image of the field from a digital camera system projected onto a $2 \mathrm{D}$ or $3 \mathrm{D}$ high-resolution monitor [11-13]. It offers high quality illumination at depth, and a broad surgical field in neuro-microvascular dissections and tumor resections [14]. One of the commonest microsurgical tasks is the microvascular anastomosis. Since there is limited experience performing microvascular anastomoses with an Exoscope [10], we have assessed whether the high-definition image, quality of magnification, and stereoscopic projection of the surgical field offer "non-inferior" value to a microsurgeon versus the standard OM.

\section{METHODS}

\section{The Modus V Exoscope}

The Modus V (Synaptive Medical Inc., 2017) is a hands-free digital microscope, with robotic arm technology based on the Canadarm device employed on the International Space Station. It incorporates a high-powered microscope, hands-free tracking of surgical instruments, automatic focus of the area of interest, pre-programming of procedural steps, and a wide range of robotic arm motion.

\section{Simulation set-up and study design}

Five expert microsurgeons, defined by performing at least one microvascular anastomosis per week, were recruited. An introductory session ensured familiarity with both the bench $\mathrm{OM}$ and the Exoscope, and an initial assessment of a single interrupted suture established a baseline performance for each. All the participants then completed a standardized arterial microvascular anastomosis with microsurgical instruments and 10/0 sutures (S\&T AG, Neuhausen, Switzerland), using both the standard bench OM (M651/M655; Leica Microsystems, Heerbrugg, Schweiz) and the Exoscope (Modus V; Synaptive Medical Inc., 2017), on fresh ex vivo chicken ischiatic arteries (estimated average vessel external diameter of $1.7 \mathrm{~mm}$ ) [15], and following a standardized bi-angulation technique (Fig. 1). The

\section{Table 1. Exoscopic devices}

\begin{tabular}{|c|c|c|c|c|}
\hline Systems & $\begin{array}{l}\text { VITOM 2D \& 3D, } \\
\text { Karl Storz. } 2011[4,5]\end{array}$ & $\begin{array}{l}\text { Computar MLH-10, } \\
\text { CBC. } 2012 \text { [6] }\end{array}$ & $\begin{array}{c}\text { ORBEYE, } \\
\text { 3D-4K video Exoscope, } \\
\text { Sony Olympus. } 2017[7,8]\end{array}$ & $\begin{array}{c}\text { Modus V } \\
\text { Synaptive Medical Inc. } 2017\end{array}$ \\
\hline \multicolumn{5}{|l|}{ Demonstration } \\
\hline Illumination & Xenon & SPD-300-W LED & $\times 2$ Fiber optic LED & Quad $(\times 4)$ LED \\
\hline Magnification (optical zoom) & $<\times 2$ & $\times 5-20$ & $<\times 6$ & $<\times 12.5$ \\
\hline Image quality & $\mathrm{HD}$ or $4 \mathrm{~K} U \mathrm{HD}$ & $H D$ & $4 \mathrm{~K}$ & $4 \mathrm{~K} U \mathrm{HD}$ \\
\hline Field of view (mm) & $50-150$ & NA & $7.5-171$ & $6.5-207.9$ \\
\hline Portability & Portable base-manual setup & Portable & Mobile base with wheels & Mobile base with castor wheel brakes \\
\hline Stereopsis-3D capability & Yes & No & Yes & Pending released (2019) \\
\hline Depth of field (mm) & $35-100$ & NA & NA & $\mathrm{X} 2$ of $\mathrm{OM}$ \\
\hline Cost (USD) & 250,000 & NA & 450,000 & $600,000-750,000$ \\
\hline
\end{tabular}




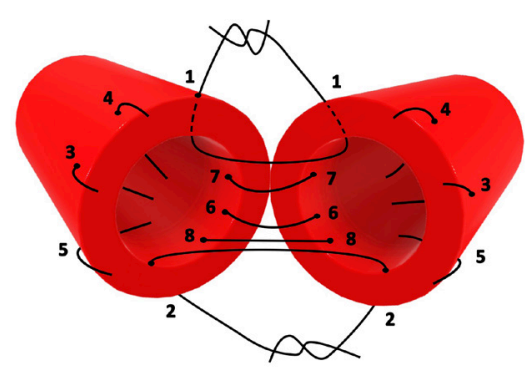

(A)

(A) The sequence of microsuture in the protocol bi-angulation technique used for microvascular anastomosis. (B) Illustration of the Modus V (Synaptive Medical Inc.) operating room setup [4]. (C) 1. Exoscope, 2. High-definition (4K) screen, 3. Hand-motion analyzer electromagnetic source, 4. Microsurgical operating field and instrumentation, 5. Hand-motion analyzer sensors. (D) System status overlay display of Modus $V$ Exoscope device with system settings appearing on video display.
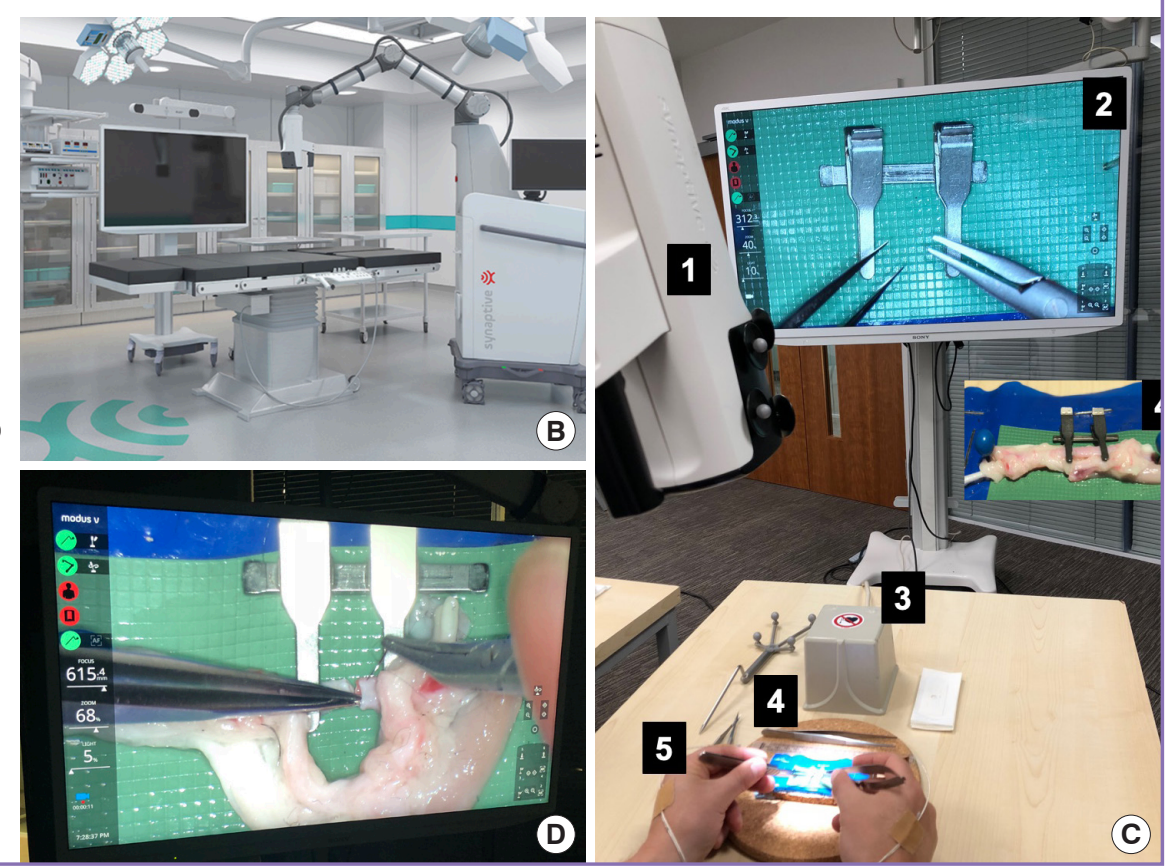

same approach provided a learning curve for a single expert performing consecutive anastomoses with the Exoscope until he reached plateau; where plateau was defined as two consecutive total procedure times with the Exoscope reaching the same or less than the time taken with the OM. Four electromagnetic sensors tracked each hand's movements within a $72 \mathrm{~cm}$ diameter electromagnetic field to evaluate surgical dexterity: one on the dorsum of each hand across the second metacarpophalangeal joint, and one on the dorsum of each index finger middle phalanx.

\section{Data collection}

The hand-motion analyzer (Dextrous MD; Inition Ltd., London, UK) provided an overall surgical performance (OSP) from measurements of the total time (TT), the total number of hand movements (TM) and the total pathlength (TP). The OSP was generated according to: $\mathrm{OSP}=\mathrm{TP} / 100$ (meters) $+\mathrm{TM} / 8$ (average movements per suture)+TT (seconds). This allowed statistical quantitative numerical comparison according to the parameters' clinical relevance. The end-product outcome from each anastomosis was assessed by the anastomosis lapse index score (ALI) [16]. The stage 0 Dextrous MD software (Inition Ltd.) processed each sensor's cartesian coordinates for each microvascular anastomosis from the first insertion of the tip of the needle, to completion of the last knot. Adverse events and major technical errors in execution and end-product anastomosis outcomes were recorded.

\section{Statistical analysis}

All data were analyzed by a blinded investigator using Microsoft Excel version 16.23 (Microsoft Corp., Redmond, WA, USA), and statistical analysis performed with GraphPad Prism version 8.0.2 (GraphPad Software, San Diego, CA, USA). Data were expressed as mean \pm standard deviation (minimum, maximum). An independent-sample t-test was used to compare $\mathrm{OM}$ and Exoscope anastomoses. A P-value $<0.05$ was considered statistically significant. Learning curves were constructed.

\section{Determination of non-inferiority margins}

The non-inferiority margin $(\Delta)$ was evaluated, based on the TT parameter, to justify the extent of clinical inferiority of the use of the Exoscope in microvascular anastomosis, provide the surgical efficiency expressed by TP and TM produced an optimal end-product (ALI score). In current literature, the average TT for a hand-sewn microvascular arterial and venous anastomosis is estimated to be 21 and 20.8 minutes, respectively [17]. The $\Delta$ margin of 10 minutes was set prospectively since a $50 \%$ increase in the TT would start to become unacceptable in terms of operating time and the financial cost to a healthcare provider. At an average cost of 20 Great British Pound (GBP) per minute for theatre time [18], an extra 200 GBP would be, for example, equivalent to the cost saving of using a coupler for a venous anastomosis, or the reasonable cost of a surgeon's break of 10 minutes while anastomotic flow was becoming clearly established following removal of the vessel clamps. 


\section{Fig. 2. OM versus Exoscope-HMA microsuture performance}

Box-plots diagrams comparison of experts' performance for: (A) total pathlength (TP), (B) total movements (TM), (C) total time (TT) performing an

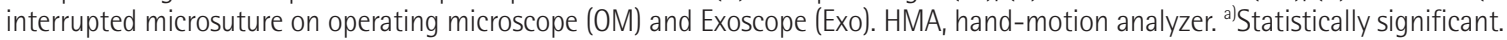
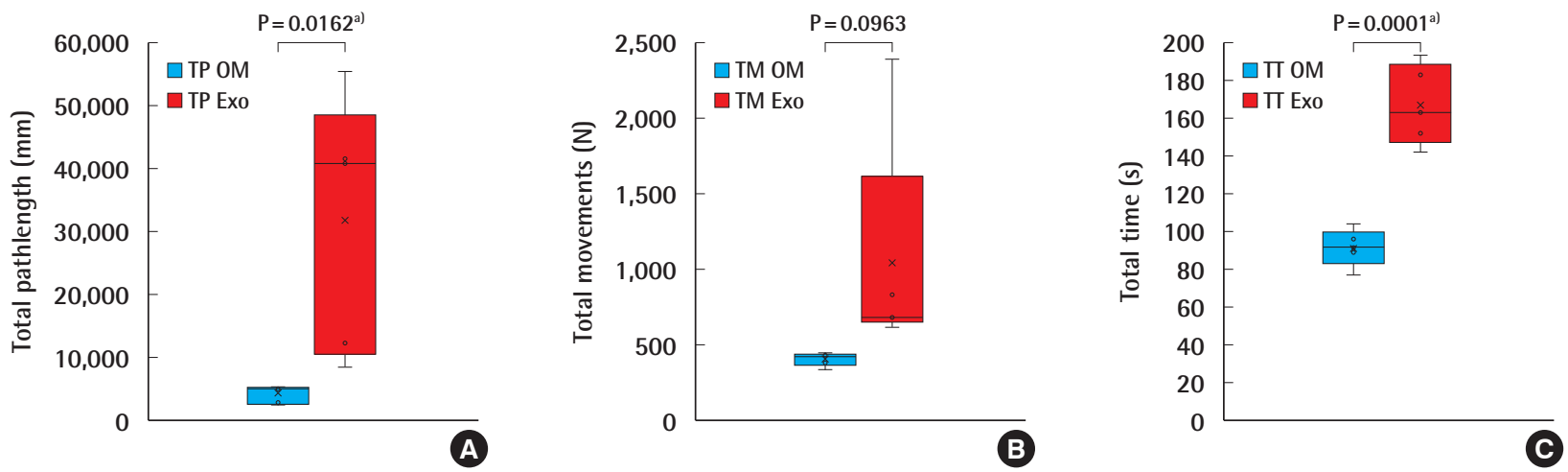

\section{Fig. 3. OM versus Exoscope-HMA anastomosis performance}

Box-plots diagrams comparison of experts' performance for: (A) total pathlength (TP), (B) total movements (TM), (C) total time (TT) performing microvascular anastomoses with the operating microscope (OM) and Exoscope (Exo). HMA, hand-motion analyzer. ${ }^{\text {a) }}$ Statistically significant.
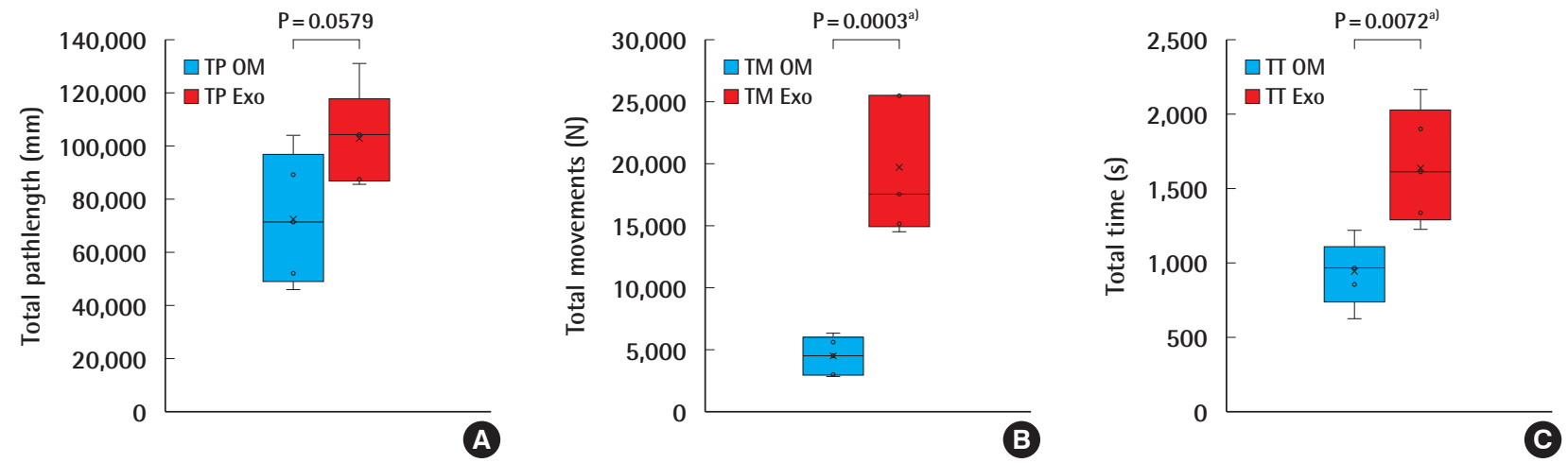

\section{RESULTS}

All the experts were assessed on completing a single interrupted suture using both the OM and the Exoscope. The TP, TM, and TT were respectively 4,029 $\pm 1,497$ (2,305, 5,358), $402 \pm 45$ $(332,446)$, and $92 \pm 10(77,104)$ for the OM, and $31,775 \pm$ 20,397 (8,504, 55,423), 1,041 $\pm 757(616,2,387)$, and $167 \pm 21$ $(142,193)$ for the Exoscope (Fig. 2). Students t-test analysis revealed statistical differences for $\mathrm{TP} ; \mathrm{t}=3.03, \mathrm{P}=0.0162$ (95\% confidence interval [CI], 6,654-48,838 mm), for TM; $\mathrm{t}=1.88$, $\mathrm{P}=0.0963$ (95\% CI, 143-1,421 movements), and for TT; $\mathrm{t}=$ 7.21, $\mathrm{P}=0.0001$ (95\% CI, 51-99 seconds).

A total of 10 anastomoses were performed, five using the $\mathrm{OM}$ and five using the Exoscope. The TP, TM, and TT for the OM and the Exoscope were $72,603 \pm 24,410$ (45,900, 103,924), $4,436 \pm 1,528(2,840,6,297), 931 \pm 219(620,1,221)$, and
$102,667 \pm 18,115$ (85,897, 130,973), 19,666 $\pm 5,493$ (14,508, $25,557), 1,645 \pm 388(1,225,2,156)$ respectively (Fig. 3$)$. Students t-test analysis revealed statistical differences for TP; $t=2.21, P=0.0579$ (95\% CI, 1,284-61,412), for TM; $t=5.97$, $\mathrm{P}=0.0003$ (95\% CI, 9,351-21,110), and for TT; $\mathrm{t}=3.59, \mathrm{P}=$ 0.0072 (95\% CI, 254-1,173). All anastomoses were evaluated for the quality of the intimal suturing, and achieved a global mean ALI score of $9.3 \pm 0.7(8,10)$; for the Exoscope and $9.6 \pm$ $0.5(9,10)$ for the OM.

A $51 \%, 58 \%$, and $46 \%$ improvement or reduction in TT, TM, $\mathrm{TP}$, respectively, during the microvascular anastomosis learning curve and reaching plateau after nine consecutive repetitions (Fig. 4). Raw inferiority in demonstrated in OSP (Fig. 5), however, clinical non-inferiority is established with inferiority margin $(\Delta)$ of 10 minutes and an absolute difference of $0.07 \mathrm{~min}$ utes between OM and Exoscope cohorts (Fig. 6). 


\section{Fig. 4. Exoscope microvascular anastomosis learning curve}

Performance after nine repetitions versus the operating microscope (OM) performance in total pathlength (TP), total time (TT), and total movements (TM) (red lines).

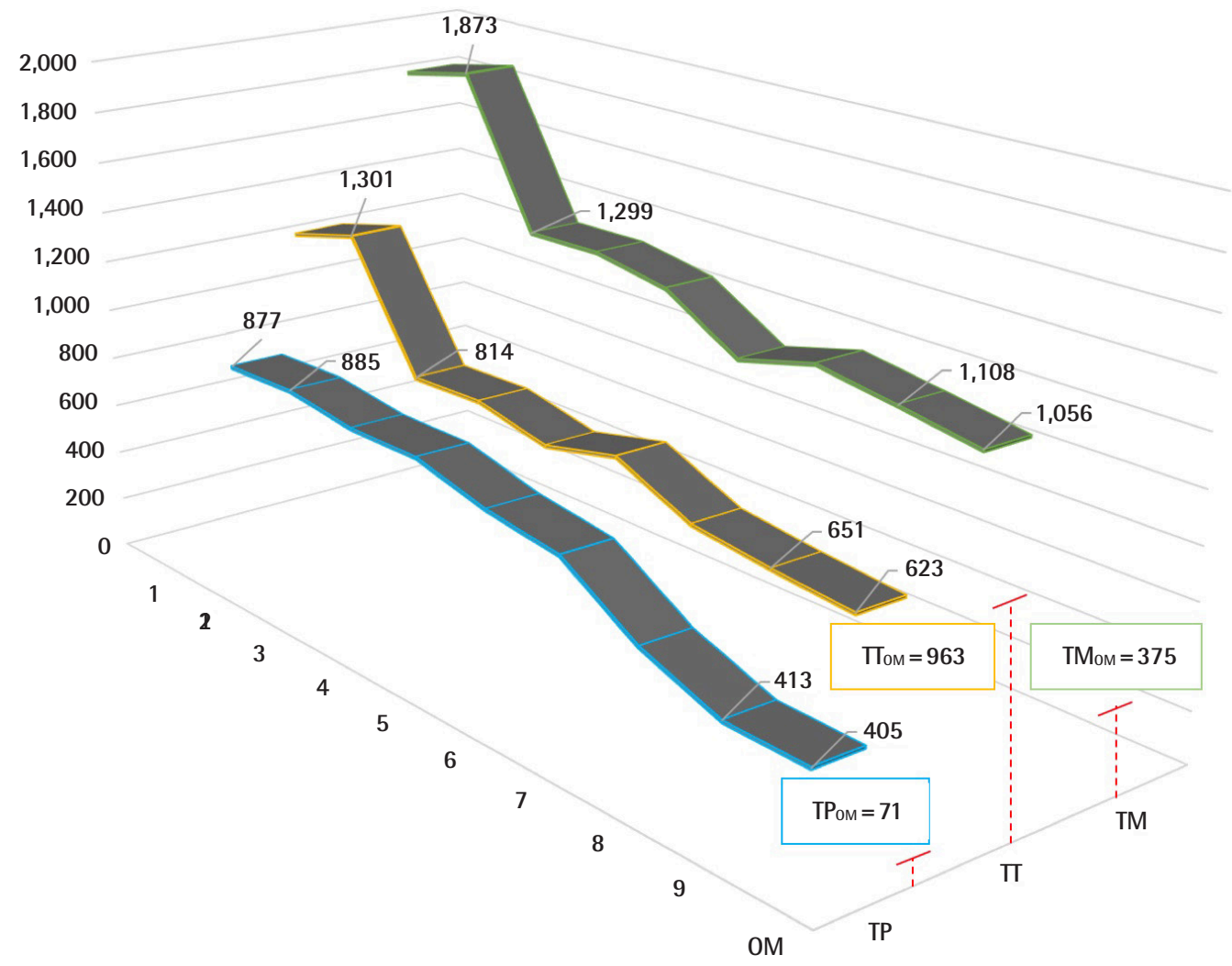

\section{Fig. 5. Overall surgical performance indicators}

Radar chart demonstrating overall surgical performance indicators for operating microscope (OM) and Exoscope.

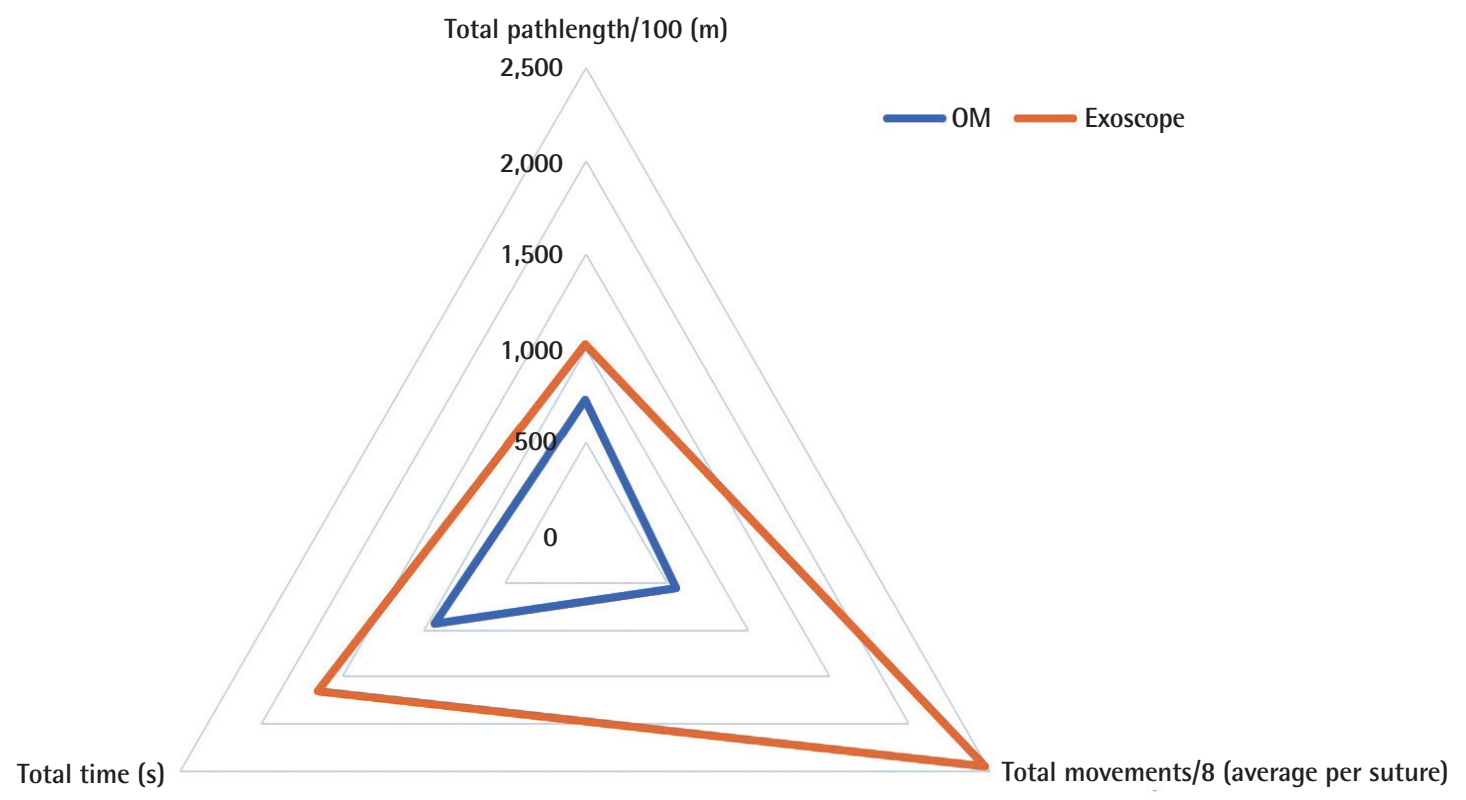




\section{Fig. 6. Non-inferiority outcomes OM versus Exoscope}

Clinical inferiority of the use of the Exoscope versus the operating microscope $(\mathrm{OM})$ in microvascular anastomosis is demonstrated with total time margin of equivalence of 10 minutes.

Total time-95\% confidence interval non-inferiority

( $\Delta$ [margin of equivalence $]=10 \mathrm{~min}$ )

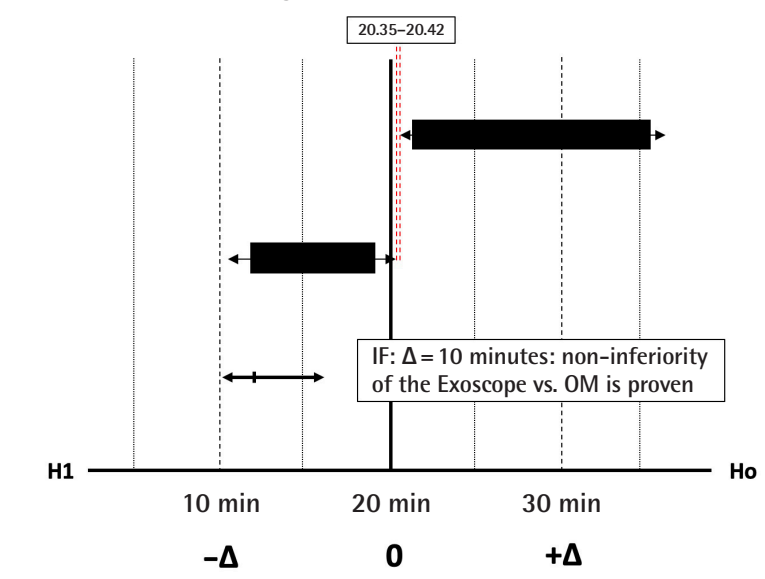

\section{DISCUSSION}

The Exoscope is a novel device offering surgical field magnification and illumination with a high-definition 2D or 3D screen image, that presents an alternative to the $\mathrm{OM}[11]$. Current literature demonstrates promise in neurosurgery [14]. The Exoscope appears at least equivalent to the OM in terms of image quality, magnification, and illumination, and offers high-resolution, precise focusing, and a broad depth of field $[19,20]$. Indeed, the Exoscope may prove superior to the OM in terms of image quality when connected to an ultra-high-definition monitor, although these advanced technical modifications could arguably be attached to any conventional OM [21]. This non-inferiority simulation study investigated the Exoscope versus the standard $\mathrm{OM}$ in the ex vivo high-fidelity microsurgical task of microvascular anastomosis, a core microsurgical skill.

This study demonstrated non-inferiority of the Exoscope versus the standard $\mathrm{OM}$ in the validated objective electromagnetic hand-motion analysis parameters TP, TM, and TT in a cohort of experts that had no prior exposure to the Exoscope. The learning curve reflected the differences in technique required to adjust from a stereoscopic $\mathrm{OM}$ to $2 \mathrm{D}$ exoscopic system, i.e., a combination of lack of stereopsis, improved image quality and magnification. We further explored and tracked the learning curve to demonstrate the "warm up" required to reach equality in the composite OSP indicator; and defined some constructive technical adjustments that may prove important in exoscopic
stereo-3D to 2D microsurgery practice [22].

Quantitative analysis of the experts' performances demonstrated that on average $28 \mathrm{~m}$ more surgical pathlength and 1.25 minutes more time were required to complete the initial single interrupted suture; and $30 \mathrm{~m}$ more surgical pathlength and 12 minutes more time to complete each anastomosis with the Exoscope, compared to the OM. These outcomes demonstrate objectively the challenging transition from traditional stereoscopic surgical vision versus stereo-3D to $2 \mathrm{D}$ screen, with a virtual digital depth perception, especially during the first attempt of the single interrupted suture. Overall, surgical performance executing the single interrupted microsuturing and microvascular endto-end anastomosis was relatively poor; but, the absolute differences in surgical pathlength and surgical time narrowed substantially and incrementally with each further suture while using novel exoscopic device. Clinically, the difference of 12 minutes in performing an arterial microvascular anastomosis in reconstructive surgery is understood to be expected to have low impact on the overall cost-efficiency of the totality of the free tissue transfer procedure [17]. Our findings, objectively support other studies reporting expert neurosurgeons using the Exoscope safely and successfully at first exposure in advanced surgical tasks [10-14,20].

The improvement in learning curve parameters, could potentially provide an early proof that Exoscope microvascular anastomosis, when performed by experts, has no negative clinical impact to the operative time (Fig. 4). These findings reinforce that a "warm up" training session can provide the surgeon with the adequate experience to overcome the technical difficulties entailing exoscopic microsurgery. Qualitative video-analysis of the anastomoses under the $\mathrm{OM}$ demonstrated bi-dexterity and fluent high-fidelity microsuturing with efficiency, agility and precision in surgical performance, whilst, the same analysis for the exoscopic performance showed adjustments in the experts' technique that facilitated safety, digital depth perception or the "see-field-feel-see" perception of surgical field.

The Exoscope appears to be safe and effective when used by an expert microsurgeon (Figs. 5, 6), and potentially offers improved ergonomics, image quality, and accessibility to the surgical field. The Exoscope has the potential to be integrated with other novel technological advancements, such as; augmented reality enhanced microsurgery and robotic-assisted microsurgery, as robotic devices that are under development require the use of a microscope to facilitate clinical performance [23]. The lack of stereopsis is its main drawback of similar video telescope operating monitor (VITOM) devices such as the endoscopicassisted microsurgery, as reported in other microsurgical subspecialties $[4,5,18,19,24]$. This can be overcome by using more 
than one $4 \mathrm{~K}$ sensors to provide a stereoscopic 3D image [25]. We visualize the future of microsurgery with advanced roboticassisted devices that synchronize in real time, while the robotic exoscopic arm follows seamlessly the micro-operative field while adjusting magnification, focus and illumination for optimal visualization. Furthermore, the motion tracking adjunct capabilities provide an easy video enhanced educational platform that could be used as a tele-medicine or tele-education platform to enhance the potentials of microsurgical education.

This study included only a limited number of participants and procedure repetitions, however, it fulfils the primary rationale and explored further the learning curve of operating advanced microvascular task stereo-3D to $2 \mathrm{D}$ screen. It also exploited the technical challenges of the lack of surgical depth and the shift from vertical to horizontal surgical orientation, which were easily manipulated by experienced surgeon to lead in safe practice with the burden of longer operating time. Further studies are required to fully characterize the learning curve and challenges of exoscopic microsurgery, while establish the level of competency and transferrable skills from OM to Exoscope operating in different clinical tasks, such as: microsurgical resections or dissections, perivascular pedicle dissection and flap elevation and microvascular advanced vessels anastomosis.

The Modus V when used with heads-up 2D stereopsis does not cause motion sickness or nausea; on the other hand, it provides an ergonomic approach to quality microsurgical operating. When the 3D glasses are used that enable 3D heads-up stereoscopic visualization; motion sickness could be claimed to be caused by excessive movements, rapid changes of the field or prolonged usage. In this study motion sickness was not observed as, in our experience during microvascular anastomosis, the field is stable, the robotic arm is used in a fixed position and the operating time is limited to approximately less than 30 minutes.

The Exoscope non-inferiority in regards to the microvascular anastomosis has been clearly investigated using objective measures (operative time, surgical efficiency/hand-motion analysis parameters) and the safety of each modality that offers magnifications (OM vs. Exoscope) has been established. Currently, initial clinical experience of this device has been demonstrated in neuro-microsurgical dissection and recent report demonstrated its applicability in flap raising and microvascular pedicle dissection including the microvascular anastomosis in free tissue transfers $[10,11]$. The VITOM technology in microvascular surgery (anastomosis), detailed the clear benefits from its innovative design, illumination and the safety of its application, including technical considerations, especially in 3D stereoscopic visualization. This study demonstrated the short learning curve in exoscopic microvascular end-to-end anastomosis, and further allows confidence and safety of the use of Exoscope in clinical environment in such a technically demanding surgical task. We believe that novel technologies that change the common practice, should be initially attempted in a safe simulation environment to allow clinical safety which cannot be universally guaranteed even in the hands of expert microsurgeons, as this may prevent errors or operating delays as a burden of its novelty.

This study demonstrated that experts' microvascular anastomosis with the Exoscope appears non-inferior to the OM. Exoscopic microvascular anastomosis was time consuming, however, the quality of the end-product (patency) was not inferior. The experts' "warm-up" learning curve is steep but swift and may prove to reach clinical equality. The expanded use of the Exoscope as an alternative to OM, in other advanced microsurgical tasks such as, perivascular, pedicle or flap dissection is yet to be objectively defined and further pre-clinical studies are required to establish its clinical efficacy and safety.

\section{NOTES}

\section{Conflict of interest}

SM has worked with Inition to develop the Dextrous MD handmotion analysis system. Synaptive Medical Inc. provided the Modus V Exoscope to the Group for Academic Plastic Surgery Group and the Microvascular Anastomosis Simulation Hub Laboratory for the period of the study data collection, however, none of the authors have conflict of interest or financial disclosures.

\section{Author contribution}

Conceptualization: G Pafitanis, S Myers. Data curation: G Pafitanis. Formal analysis: G Pafitanis. Methodology: G Pafitanis, M Hadjiandreou, S Myers. Project administration: G Pafitanis. Visualization: G Pafitanis. Writing - original draft: G Pafitanis, $M$ Hadjiandreou. Writing - review \& editing: G Pafitanis, $M$ Hadjiandreou, A Alamri, C Uff, D Walsh, S Myers.

\section{ORCID}

Georgios Pafitanis https://orcid.org/0000-0003-4712-277X Michalis Hadjiandreou

https://orcid.org/0000-0002-2535-7896

\section{REFERENCES}

1. Jacobson JH 2nd, Wallman LJ, Schumacher GA, et al. Microsurgery as an aid to middle cerebral artery endarterectomy. J Neurosurg 1962;19:108-15.

2. Acland R. Signs of patency in small vessel anastomosis. Sur- 
gery 1972;72:744-8.

3. Koshima I, Yamamoto T, Narushima M, et al. Perforator flaps and supermicrosurgery. Clin Plast Surg 2010;37:683-9.

4. Nishiyama K. From exoscope into the next generation. J Korean Neurosurg Soc 2017;60:289-93.

5. Robert E, Facca S, Atik T, et al. Vascular microanastomosis through an endoscopic approach: feasibility study on two cadaver forearms. Chir Main 2013;32:136-40.

6. Zhu X, Luo J, Liu Y, et al. Screen-imaging guidance using a modified portable video macroscope for middle cerebral artery occlusion. Neural Regen Res 2012;7:912-6.

7. Sack J, Steinberg JA, Rennert RC, et al. Initial experience using a high-definition 3-dimensional exoscope system for microneurosurgery. Oper Neurosurg (Hagerstown) 2018;14: 395-401.

8. Olympus Corp. Launch of ORBEYE surgical microscope with 4K 3D capabilities [Internet]. Tokyo: Olympus Corp.; c2017 [cited 2020 May 8]. Available from: https://www. olympus-global.com/news/2017/nr00611.html?page=ir.

9. Cheng HT, Ma H, Tsai CH, et al. A three-dimensional stereoscopic monitor system in microscopic vascular anastomosis. Microsurgery 2012;32:571-4.

10. Piatkowski AA, Keuter XHA, Schols RM, et al. Potential of performing a microvascular free flap reconstruction using solely a 3D exoscope instead of a conventional microscope. J Plast Reconstr Aesthet Surg 2018;71:1664-78.

11. Mamelak AN, Danielpour M, Black KL, et al. A high-definition exoscope system for neurosurgery and other microsurgical disciplines: preliminary report. Surg Innov 2008;15: 38-46.

12. Oertel JM, Burkhardt BW. VITOM-3D for exoscopic neurosurgery: initial experience in cranial and spinal procedures. World Neurosurg 2017;105:153-62.

13. Rossini Z, Cardia A, Milani D, et al. VITOM 3D: preliminary experience in cranial surgery. World Neurosurg 2017; 107:663-8.

14. Ricciardi L, Chaichana KL, Cardia A, et al. The exoscope in neurosurgery: an innovative "point of view". A systematic review of the technical, surgical and educational aspects. World Neurosurg 2019;124:136-44.

15. Kang BY, Jeon BJ, Lee KT, et al. Comprehensive analysis of chicken vessels as microvascular anastomosis training model. Arch Plast Surg 2017;44:12-8.

16. Ghanem AM, Al Omran Y, Shatta B, et al. Anastomosis lapse index (ALI): a validated end product assessment tool for simulation microsurgery training. J Reconstr Microsurg 2016;32:233-41.

17. Fitzgerald O'Connor E, Rozen WM, Chowdhry M, et al. The microvascular anastomotic coupler for venous anastomoses in free flap breast reconstruction improves outcomes. Gland Surg 2016;5:88-92.

18. Institution for Innovation and Improvement. Improving quality and efficiency in the operating theatre [Internet]. Coventry: NHS; c2019 [cited 2020 Apr 13]. Available from: http://harmfreecare.org/wp-content/files_mf/Improvingquality-and-efficiency-in-the-operating-theatre.pdf.

19. Krishnan KG, Scholler K, Uhl E. Application of a compact high-definition exoscope for illumination and magnification in high-precision surgical procedures. World Neurosurg 2017;97:652-60.

20. Shirzadi A, Mukherjee D, Drazin DG, et al. Use of the video telescope operating monitor (VITOM) as an alternative to the operating microscope in spine surgery. Spine (Phila Pa 1976) 2012;37:E1517-23.

21. Moisi MD, Hoang K, Tubbs RS, et al. Advancement of surgical visualization methods: comparison study between traditional microscopic surgery and a novel robotic optoelectronic visualization tool for spinal surgery. World Neurosurg 2017;98:273-7.

22. Chan W, Niranjan N, Ramakrishnan V. Structured assessment of microsurgery skills in the clinical setting. J Plast Reconstr Aesthet Surg 2010;63:1329-34.

23. van Mulken TJM, Boymans CAEM, Schols RM, et al. Preclinical experience using a new robotic system created for microsurgery. Plast Reconstr Surg 2018;142:1367-76.

24. Birch K, Drazin D, Black KL, et al. Clinical experience with a high definition exoscope system for surgery of pineal region lesions. J Clin Neurosci 2014;21:1245-9.

25. Beez T, Munoz-Bendix C, Beseoglu K, et al. First clinical applications of a high-definition three-dimensional exoscope in pediatric neurosurgery. Cureus 2018;10:e2108. 